\title{
Juxtaposing Vertically Transmitted Infections (VTIs) and the Spread of HIV/AIDS in a Typically Infection Prevalent Region in Nigeria
}

\author{
Matthew Iseh, Anthony Usoro, Nsisong Ekong*, Idara Ukpe \\ ${ }^{a}$ Department of Statistics, Akwa Ibom State University, Ikot Akpaden, Nigeria
}

\begin{abstract}
In this paper, we investigate by putting side by side the rate at which Human Immuno-deficiency Virus/ Acquired Immune Deficiency Syndrome HIV/AIDS spreads through Vertical Transmission (VT) in a typically infection prevalent region. Also, we model this relationship and present the enormity and the likelihood of mother-to-child infections given Age, Weight, and Dosage of Drugs taken by the pregnant women. Binary logistics regression is the approach employed in the analysis of this work. The method is necessary given the dichotomous nature of the variable under investigation, in this case, the status of the newborns. The result from the Binary logistic regression shows that the probability that a child will be infected given that the mother is positive is $0.098235 \approx 0.1$, and this is unlikely to happen given the small probability. This result is in line with the proportion of Event count which is $\approx 0.1$. Other complimentary test results also agree that given the biomarkers (Age, weight and drug doses of the mothers) used in the study, the possibility of a newborn acquiring the deadly disease is very minimal. The study revealed that, given these biomarkers, the chances of newborns being infected with HIV/AIDS through VT is very minimal. Hence, there is need for donors and interventionists agencies to redirect attention and interventions to other modes of transmissions.
\end{abstract}

DOI:10.46481/jnsps.2022.418

Keywords: Human Immunodeficiency Virus (HIV), Acquired Immune Deficiency Syndrome (AIDS), Vertically Transmitted Infections (VTIs), Akwa Ibom State.

Article History :

Received: 20 September 2021

Received in revised form: 26 November 2021

Accepted for publication: 02 January 2022

Published: 28 February 2022

(c)2022 Journal of the Nigerian Society of Physical Sciences. All rights reserved. Communicated by: W. A. Yahya

\section{Introduction}

The speedy upsurge of certain diseases notwithstanding the myriads of precautionary and preventive measures taken globally by different quarters to evade the prevalence of such diseases is a thing of great concern. One of such diseases is the Human Immuno-deficiency Virus/ Acquired Immune Deficiency Syndrome HIV/AIDS - a disease that is still considered a major global public health issue. So far, it has been with us for

${ }^{*}$ Corresponding author tel. no: +2347069150513

Email address: nsisongekong@aksu.edu.ng (Nsisong Ekong) the past six decades and has so far claimed not less than 33 million lives globally [9]. According to the same report, it is estimated that 38 million people were living with this disease at the end of 2019 across the globe. Upon an establishment that the disease is one of the numerous Vertically Transmitted Infections (VTIs), that is, the transmission of from a mother to her child during pregnancy, labour, delivery or breastfeeding, different approaches have been set in motion to curb the mother-to-child transmission and so far, about $85 \%$ of either pregnant women or breastfeeding mothers living with the disease have received Anti-Retroviral Therapy (ART) which apart 
from reducing the possibilities of transmitting this disease to their newborns, has made the disease manageable thereby enabling the carriers to lead long and healthy lives. Unlike other forms of infection, preventing vertical transmission of HIV is more complex, requiring intervention from preconception to the end of breastfeeding. This includes primary prevention of HIV before, during, and after pregnancy; prevention of unwanted or unplanned pregnancies; early initiation of ART; retention on ART for women with HIV; and retesting women without HIV to detect new infections early. Early antenatal care is also important to optimise general care, monitoring of maternal viral load, safe delivery practices, and infant postexposure prophylaxis [10]. Though a good number of women within this subgroup have had good access to these therapies and interventions, others are getting it difficult, or at worst, not able to access it at all. Several factors may contribute to this vulnerability. It could be legal, religious, illiteracy, biological, social, or sociocultural ([1]; [8]). In the absence of any intervention, transmission rates range from $15 \%$ to $45 \%$ ([4]; [3]). This rate can be reduced to below $5 \%$ with effective interventions during the periods of pregnancy, labour, delivery and breastfeeding. These interventions primarily involve antiretroviral treatment for the mother and a short course of antiretroviral drugs for the baby. They also include measures to prevent HIV acquisition in the pregnant woman and appropriate breastfeeding practices.

However, a concerted global effort against this disease has steadily increased the HIV services coverage. Apart from the administration of $68 \%$ adults and $53 \%$ of children living with HIV globally, one of such efforts is the move to eradicate mother to child transmission of the disease. This genuine interest to curb further spread in the West African region also gave birth to Nigerian HIV/AIDS Indicator and Impact Survey (NAIIS) in 2018 - a collaborative project by Federal Ministry of Health, Nigeria, University of Baltimore, Maryland, etc. in attempt to ascertain the prevalence of the disease in the country. The report of this survey as published on NAIIS website and other media houses showed that Akwa Ibom State, the major oilproducing state in the country, has the highest number of people living with this virus. Though the survey and data collection approach used was best known to the investigators, the report, however, raises some sort of panic amongst the residents of the state. Nevertheless, this panic is neither peculiar to Akwa Ibom State nor Nigeria, but to other African sub-region and the world at large. To understand the prevalence trend and other infection dynamics of the disease in this state, a good number of researches have been carried out on this topic. To proffer ways of reducing the Mother-to Child-Transmission (MTCT), [2] carried out some findings from an early infant diagnosis program in the south-south region of Nigeria (with Akwa Ibom state inclusive) and concluded that reduction of MTCT of HIV is possible with effective Prevention of Mother-to-Child Transmision (PMTCT) interventions, including improved access to ARVs for PMTCT and appropriate infant feeding practices. On the same vein, [5] investigated knowledge and practice of prevention of Mother-To-Child-Transmission of HIV among traditional birth attendants practising in the state, and they discovered that the knowledge and practice of PMTCT of HIV by the TBAs practising in Akwa Ibom is less than adequate thus increasing the risk of vertical transmission of HIV. The authors suggested an urgent need for the education and training of TBAs to improve their knowledge and enhance their practice. On the global scene, many pieces of research are carried out on this topic and they could be found in the literature ([7]; [6]; and others).

Different from these researches which entirely focus on the preventive measures on tackling mother-to-child-transmission, this paper will employ the technique of logistic regression to give an insight into the proportion of HIV/AIDS infections attributed to VT in Akwa Ibom State. Based on the data available, the paper will predict the status of newborns given the chosen biomarkers in the pregnant and breastfeeding women. This paper is organised as follows; in section 1, a general introduction is given, section 2 gives an overview of the methodology employed in the data analysis, and section 3 presents the results of the analyses. Finally, section 4 concludes with a summary.

\section{Methodology}

In the analysis of data related to this research, we will employ the Binary Logistic Regression technique.

\subsection{Logistic Regression Analysis}

Regression methods have become an integral component of any data analysis when it comes to deciphering the relationship between a response variable and explanatory variable(s) given that the dataset are discrete in nature. Logistic Regression Model has become the standard of analysis in many fields of studies specifically the Medical field. At this point it is pertinent to note that the goal of Logistic Regression is to find the best fitting and closest, yet reasonable model to describe the affinity or relationship between an outcome that is dependent or response variable and a set of independent otherwise known as predictor or explanatory variables. In Statistics, Logistic Regression, or Logit Regression, or Logit Model is a regression model where the dependent variables are categorical. Unlike the Linear Regression Analysis which is continuous, it is binary or dichotomous in nature, also the choice of a parametric model and assumptions differs, thus, these features distinguish it from others. It could be noted that once this difference has been accounted for, the procedures employed in an analysis of logistic regression follows the same general principle of that of the linear regression.

\subsubsection{The Odds}

Consider the Linear Regression for $n$-independent variables as follows

$$
Y=\beta_{0}+\beta_{1} X_{1}+\beta_{2} X_{2}+\ldots+\beta_{n} X_{n}+\varepsilon
$$

where $Y$ is the response variable, $X_{1}, X_{2}, \ldots, X_{n}$ are the predictor variables with associated parameters $\beta_{1} \beta_{2}, \ldots, \beta_{n}$ respectively. $\varepsilon$ is the error term associated with the model. 
The above equation could be considered as Binomial case with $\mathrm{n}$-trials concerning the response variable. Also, consider the Linear Regression with three independent variables we have;

$$
\hat{Y}=\hat{\beta}_{0}+\hat{\beta}_{1} X_{1}+\hat{\beta}_{2} X_{2}+\hat{\beta}_{3} X_{3}+\varepsilon
$$

In the above equation, $X_{1}, X_{2}$, and $X_{3}$ are the biomarkers representing the Age of the infected pregnant women, the Weight of the pregnant women infected, and the drug dosage taken by the pregnant women infected respectively.

$\mathrm{Eq}(2)$ is the Bernoulli distribution with $n=1$ with respect to the dependent variable $Y$, which represent the Binary Logistic Regression with $n=1$ trials, where Success is "1" and failure is "0" Also, recall that the probability of Success in a Bernoulli Distribution is $p$ and failure $q$ is given as follows:

$$
q=1-p
$$

Recall that, the Expectation of a Bernoulli Distribution, given as $E(X)$ is

$$
E(X)=\frac{p}{q}
$$

For $q=1-p$, Eq. (3) becomes

$$
E(X)=\frac{p}{1-p}
$$

Eq. (3) is called the odds, which is the expected value of a random variable having a Bernuoulli distribution. In logistic regression, we estimate an unknown $p$ for any given linear combination of the independent variables, thus, linking the independent variables to the Bernoulli distribution gives us the $\operatorname{Logit}(l)$. This, of course, is the ultimate goal of the Logistic Regression. Thus, we need a function that links the linear combination of variables and the Bernoulli distribution together or map the linear combination of variables that could result in any value onto the Bernoulli probability distribution with a domain from 0 to 1 . This result in taking the natural $\log$ of Eq.(4), which is the log of the odds. Thus this gives;

$$
\operatorname{Logit}(l)=\operatorname{Logodd} s=\ln \left(\frac{p}{1-p}\right)=\hat{Y}
$$

\subsubsection{The Binary Logistic Regression}

The Binary logistic regression model is obtained from the combination of Eqs. (2) and (5). So that we have,

$$
\ln \left(\frac{p}{1-p}\right)=\hat{\beta}_{0}+\hat{\beta}_{1} X_{1}+\hat{\beta}_{2} X_{2}+\hat{\beta}_{3} X_{3}
$$

Taking the exponent of Eq. (6) above, we have

$$
\frac{p}{1-p}=e^{\hat{\beta}_{0}+\hat{\beta}_{1} X_{1}+\hat{\beta}_{2} X_{2}+\hat{\beta}_{3} X_{3}}
$$

By simplification, we obtain

$$
\hat{p}=\frac{e^{\hat{\beta}_{0}+\hat{\beta}_{1} X_{1}+\hat{\beta}_{2} X_{2}+\hat{\beta}_{3} X_{3}}}{1+e^{\hat{\beta}_{0}+\hat{\beta}_{1} X_{1}+\hat{\beta}_{2} X_{2}+\hat{\beta}_{3} X_{3}}}
$$

Eq. (8) gives the estimate of the Logistic Regression, where $X_{1}, X_{2}$, and $X_{3}$ are as defined earlier with their respective estimated parameters $\hat{\beta_{1}}, \hat{\beta_{2}}$, and $\hat{\beta_{3}}$, e denotes the exponential function.

$p$ is the probability that the child is infected given the mother has the disease.

\subsubsection{The Odds Ratio}

The odds ratio (denoted as $\theta$ ) between the odds of two sets of the predictor (say $X_{1} X_{2}$ ). For a continuous independent variable, the odds ratio defined as:

$$
\theta=\frac{\frac{p}{(1-p)} \| X=X_{1}}{\frac{p}{(1-p)} \| X=X_{2}}
$$

But the odds of success are as given in (7),

\subsubsection{Wald Test}

The Wald test (also called the Wald Chi-Squared Test) is a way to find out if explanatory variables in a model are significant. "Significant" means that they add something to the model; variables which add nothing is removed without affecting the model in any meaningful way. The test can be used for a multitude of different models, including those with binary variables or continuous variables. The null hypothesis for the test is: some parametesr are zero in value (not sugnificant). If the null hypothesis is rejected, it suggests that the variables in question can be removed without much harm to the model fit. If the Wald test shows that the parameters of some explanatory variables are zero, you can remove the variables from the model. If the test shows the parameters are not zero, you should include the variables in the model. The Wald test statistic formula is:

$$
W a l d \operatorname{Test}\left(W_{j}\right)=\frac{\beta_{j}^{2}}{S E\left(\beta_{j}^{2}\right)}
$$

where; $\beta_{j}^{2}$ is the square of the linear regression coefficient, $S E\left(\beta_{j}^{2}\right)$ is the square of the standard error of the regression coefficient and is asymptotically distributed as a chi-square distribution with 1 degree of freedom.

\subsubsection{The Likelihood Function for Logistic Regression (De- viance)}

Deviance measures the discrepancy between the current model and the full model. The full model is the model that has $\mathrm{n}$ parameters, one parameter per observation. The full model maximizes the log-likelihood function. The full model provides a point of comparison for reduced model, that is, model with fewer than n parameters. Comparisons to the full model use the scaled deviance. The contribution to the scaled deviance from each individual data point depends on the model. The likelihood is given in the equation below

$$
\Lambda^{*}=-2\left(l\left(\beta^{0}\right)-l\left(\beta^{1}\right)\right)
$$

where $l\left(\beta^{1}\right)$ is the log-likelihood of the fitted (full) model. $l\left(\beta^{0}\right)$ is the log-likelihood of the (reduced) model specified by the null hypothesis evaluated at the maximum likelihood estimate of that reduced model.

\section{Data Analysis and Results}

$\mathrm{R}$ and Minitab were used in the analysis of the recorded cases of vertically transmitted HIV/AIDS in Akwa Ibom State. 
Table 1: 2018 Early Infant Diagnosis (EID) Results Summaro

\begin{tabular}{ccccc}
\hline \hline Mnth & No. of PW & No. of + & Enrolled & Not Enrolled \\
\hline Jan & 291 & 23 & 16 & 7 \\
Feb & 48 & 15 & 12 & 3 \\
Mar & 193 & 12 & 9 & 3 \\
Apr & 200 & 13 & 11 & 2 \\
May & 89 & 20 & 13 & 7 \\
Jun & 36 & 12 & 10 & 2 \\
Jul & 132 & 33 & 16 & 17 \\
Aug & 96 & 12 & 10 & 2 \\
Sep & 143 & 9 & 7 & 2 \\
Oct & 92 & 5 & 0 & 5 \\
Nov & 250 & 12 & 11 & 1 \\
Total & 1570 & 166 & 115 & 51 \\
\hline
\end{tabular}

Table 2: Data Count

\begin{tabular}{ccc}
\hline \hline Variable & Value & Count \\
\hline $\mathrm{Y}$ & 1 & 166 (Event) \\
& 0 & 1404 \\
& Total & 1570 \\
\hline
\end{tabular}

Table 3: Model Summary

\begin{tabular}{ccc}
\hline \hline Deviance & Deviance & \\
\hline R-Sq & R-Sq (adj) & AIC \\
$0.04 \%$ & $0.00 \%$ & 1067.35 \\
\hline
\end{tabular}

\subsection{Data Collection}

Data used for this research work are secondary data obtained from the Akwa Ibom State Ministry of Health (MOH). Monthly dataset for the year 2018 was considered.

\subsection{Data Presentation}

Table 1 shows the dataset used for the analysis of this research, $P W$ : Pregnant Women.

+ : Positive.

Mnth: Month

Enrolled: Number of pregnant women on Antiretroviral Therapy (ART) intervention. Not Enrolled: Number of pregnant women not on Antiretroviral Therapy (ART) intervention.

\subsection{Results of Analyses}

The data was Bernoulli (binary) coded to reflect the status of the respondents. The data were randomly distributed without any prior knowledge about the outcome The data count is as shown in Table 2.

The outputs are given in Table 4: Binary Logistic Regression: Y(newborn HIV/AIDS status-Positive (1), Negative (0) versus Age, Weight, Dosage (Drugs)
3.4. The Binary Logistic Regression Function or the Logit

Recall that the Logit Function is as given below.

$$
P=e^{\hat{y}}
$$

where

$$
\hat{y}=\hat{\beta}_{0}+\hat{\beta}_{1} X_{1}+\hat{\beta}_{2} X_{2}+\hat{\beta}_{3} X_{3}
$$

Using the above, we have that

$\hat{Y}_{i}=-2.312+0.0037$ Age -0.121 Weight +0.314 Dosage $($ Drugs $)$

The probability of the child being infected is computed as follows

$$
p=\frac{e^{\hat{Y}}}{\left(1+e^{\hat{Y}}\right)}
$$

For $i=1$, the probability of that child being positive given that the mother is negative is given by;

$$
p=\frac{e^{\hat{Y}}}{1+e^{\hat{Y}}}=\frac{0.108937}{1+e^{(-2.21699)}}=0.098235
$$

Thus, the probability that her child will be infected is 0.098235 $\approx 0.1$, and this is unlikely to happen given the small probability. This result agrees with the proportion of Event count recorded in Table 2 as $\frac{166}{1570} \approx 0.1$

\subsection{The Odds Ratio}

The odds ratio denoted by $\theta$ and given in Eq. (10) is the odds ratio between two sets of the predictor presented in Table 5 below.

The result above implies that a unit increase in Age increases the odds of a newborn being infected by the mother by a factor of 1.0037 . In the same vein, the result shows that a unit increase in Weight increases the odds of a newborn being infected by the mother by a factor of 0.8862 . And also, a unit increase in Dosage of Drugs increases the odds of a newborn being infected by the mother by a factor of 1.3691 . This follows that Age and Dosage may increase the odds of a newborn being infected by an infected mother minimally while Weight does not yeild such increase at all. 
Table 4: Coefficients (Wgt:Weight; DD: Drug Dosage.)

\begin{tabular}{ccccccc}
\hline \hline Term & Coef & SE & $95 \%$ CI & Z-Val & P & VIF \\
\hline Const & -2.312 & 0.503 & $(-3.298,-1.326)$ & -4.59 & 0 & \\
Age & 0.004 & 0.011 & $(-0.018,0.025)$ & 0.34 & 0.733 & 1 \\
Wgt & -0.121 & 0.550 & $(-1.199,0.958)$ & -0.22 & 0.826 & 1 \\
DD & 0.314 & 0.651 & $(-0.962,1.590)$ & 0.48 & 0.629 & 1 \\
\hline
\end{tabular}

Table 5: Odds ratio

\begin{tabular}{ccc}
\hline \hline & Odds Ratio & $95 \%$ CI \\
\hline Age & 1.0037 & $(0.9824,1.0255)$ \\
Weight & 0.8862 & $(0.3014,2.6056)$ \\
Dosage (Drugs) & 1.3691 & $(0.3821,4.9056)$ \\
\hline
\end{tabular}

\subsection{The Wald Test}

The Wald test is the test of Significance for individual regression coefficients in the Logistic Regression, (recall that in the Generalized Linear Regression Analysis we use the t-test statistics). For maximum likelihood estimates, we use Eq. (11), and these are $z$-values given in Table 4 .

Using the formula in Eq. (10) and the values of $\beta^{\prime} s$, the Wald test is computed and the result is as follows, 0.113 for Age, 0.0484 for Weight and 0.233 for Drugs Dosage. The smallness of the value of the Wald Test result for the Weight biomarker suggests that the presence of Weight may not be necessary for the model. The above indicator informs us to refit the model without the Weight; the parameters of the reduced model are presented in Table 6;

The Wald test results for the two biomarkers are 0.116 for Age and 0.225 for Dosage of Drugs. These may not be as strong as should, but it is a better model to be entertained.

\subsection{The Likelihood Ratio Test or Deviance}

Here, we use the likelihood-ratio test to compare two models. Specifically, we are testing $H_{0}: \beta_{\text {Age }}=\beta_{\text {Weight }}=\beta_{\text {Dosage }}=$ 0 The Likelihood ratio test is used to test the null hypothesis $\left(H_{0}\right)$ that any subset of the $\beta^{\prime} s=0$. Where the number of $\beta^{\prime} s=0$ in the full model is $p$, while the number of the $\beta^{\prime} s=0$ in the reduced model is $r$. ( $r$ is the model that results when $\beta^{\prime} s=0$ in the null hypothesis are set to 0$)$. The result is as given in the Analysis of Deviance in Table 7. From the result of our Wald Test, it is observed that the value of the test for Weight was small. At that point, it was suspected that fitting a new model without the variable would give a better fit. Due to this, a reduced model with Weight was fitted and the Deviance analysis carried out.

We see that the test statistic that we had just calculated appears in the output. The very big p-value suggests that there is no significant difference between the larger model and the reduced one. The values of the residual deviance for the two models also support the above claim as the two values are almost the same. Hence, there is need for the Weight biomarker to be removed from the model since it may lead to overfitting. Of course, this is also an indication that in investigating the VT infections from positive pregnant women to the newborns, the weight of the women is not necessarily one of the determining factors. An overview of the two fitted models generally suggests that Age, Weight, and Dosage of drugs taken by reactive pregnant women do not imply or contribute to the vertical transmission of the HIV/AIDS from the mothers to their newborns.

\section{Conclusion}

This paper is designed to explore the proportion of HIV/AIDS infections attributed to VT in Akwa Ibom State. From the data collected from the Akwa Ibom Ministry of Health on the virus and VT related cases, the paper used the Binary Logistic Regression Analysis to predict the HIV status of newborns given the chosen biomarkers in the pregnant and breastfeeding women, in this case, Age, Weight, and Drug (Dosage) intake. In doing this, the Logit function, the Odds ratio, the Wald test, and the Likelihood Ratio test were used to explain the adequacy of the fitted model. The results clearly showed that given these biomarkers (Age, Weight, and Dosage of Drugs), the rate of spread of the HIV/AIDS is seldom through vertical transmission, that is, from an infected mother to her newborn. The implication of this finding is that the prevalence rate of the disease in Akwa Ibom State may be attributed more to other modes of transmission and less to VT. While it is important to continue in the interventions focusing on antiretroviral treatments for the positive mothers, it is more advisable to intervene by creating more awareness on the other modes of transmission.

\section{Abbreviations}

HIV - Human Immunodeficiency Virus.

AIDS- Acquired Immune Deficiency Syndrome.

VTIs - Vertically Transmitted Infections.

VT - Vertical Transmision.

MTCT - Mother-To-Child Transmision.

PMTCT - Prevention of Mother-to-Child Transmision.

NAIIS - Nigeria AIDS/HIV Indicator and Impact Survey. ART - Antiretroviral Therapy.

\section{Availability of data and materials}

All data used for supporting the conclusions of this article are available from the public data repository at the website https://zenodo.org/record/4243862. 
Table 6: Coefficient of the reduced model

\begin{tabular}{ccccc}
\hline \hline & Estimate & Std. Error & $\mathrm{t}$ value & $\operatorname{Pr}(>|t|)$ \\
\hline (Intercept) & -2.373300 & 0.418955 & -5.665 & $1.75 \mathrm{e}-08$ \\
Age & 0.003736 & 0.010968 & 0.341 & 0.733 \\
Dosage of Drugs & 0.308619 & 0.650987 & 0.474 & 0.636 \\
\hline \hline
\end{tabular}

Table 7: Analysis of deviance Table

\begin{tabular}{cccccc}
\hline \hline & Resid. Df & Resid. Dev & Df & Deviance & $\operatorname{Pr}(>$ Chi $)$ \\
\hline 1 & 1566 & 1059.3 & & & \\
2 & 1567 & 1059.4 & -1 & -0.048187 & 0.8265 \\
\hline \hline
\end{tabular}

\section{References}

[1] E. A. Anigilaje, O. J. Dabit, B. Ageda, S. Hwande, T. T. Bitto, "The prevalence and predictors of HIV infection among children of mothers who missed prevention of mother to child transmission of HIV interventions in Makurdi, Nigeria", J AIDS Clin Res. 4 (2013) 249.

[2] C. Anoje, B. Aiyenigba, C. Susuki,T. Badru, K. Akpoigbe, M. Odo, S. Odafe, O. Adedokun, K. Torpey and O. N. Chabikuli, "Reducing Motherto-Child transmission of HIV: Finding from an early infant diagnosis program in South-South Nigeria" BMC Public Health 12 (2012) 184.

[3] Besser, M. HIV In Pregnancy: Doing More with Less: Mothers2Mothers (2010).

[4] E. M. Connor, R. S. Sperling, R. Gelber, P. Kiselev, G. Scott, M. J. O'sullivan, R. VanDyke, M. Bey, W. Shearer, R. L. Jacobson \& E. Jimenez, "Reduction of maternal-infant transmission of human immun- odeficiency virus type 1 with zidovudine treatment", New England Journal of Medicine 331 (1994) 1173

[5] U. I. Eshiet, \& P. G. Ekpe, "Knowledge and Practice of Prevention of Mother-to-Child- Transmission of HIV among Traditional Birth Attendants Practicing in Akwa Ibom State, Nigeria”, British Journal of Pharmaceutical Research 13 (2016) 10.

[6] N. P. Nyoyoko, A. V. Umoh, "The Prevalence and Determinants of HIV Seroconversion among ante natal clients in the University of Uyo Teaching Hospital, Uyo, Akwa State, Nigeria”, Pan Afro. Med. J 25 (2016) 247.

[7] M. Newell, "Prevention of Mother-to-Child-Transmission of HIV: Challenge for the current decade", Bulletin of the World Health Organization 200179 (2001) 12.

[8] B. O. Ogunbosi, R. E. Oladokun, O. Awolude, B. J. Brown, O. A. Adeshina, M. Kuti, B. Taiwo, B. Berzins, D. N. Kyriacou, E. G. Chadwick and K. Osinusi, "Missed opportunities for prevention of mother-to-child transmission of HIV (PMTCT) in Ibadan, Southwest Nigeria", World J AIDS 4 (2014) 356. http://dx.doi.org/10.4236/wja.2014.43042

[9] World Health Organization HIV/AIDS Key Facts. www.who.int/newsroom/facts-sheets/detail/hiv-aids, (2020)

[10] World Health Organization. Use of antiretroviral drugs for treating pregnant women and preventing HIV infection in infants. https://www.who.int/hiv/pub/mtct/programmatic_update2012/en/ (2012) 